\title{
Is Not It the Time to Change the Treatment of Intermediate-Risk Patients Suffering From Gestational Trophoblastic Neoplasia?
}

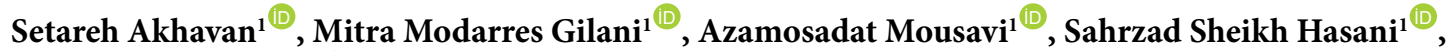 \\ Fahimeh Nokhostin ${ }^{2 *}$
}

\begin{abstract}
Objectives: The present study attempted to provide a clear view of gestational trophoblastic neoplasia (GTN) with the focus on resistance to treatment approaches in Iran.

Materials and Methods: This retrospective cohort study reviewed the medical records of 272 patients with the definitive diagnosis of GTN referring to Imam Khomeini hospital in Tehran during 2007-2017.

Results: The mean age of participants was $29.19 \pm 7.46$ years. The abnormal uterine bleeding (AUB) was the most common clinical manifestation in $64.3 \%$ of patients. Regarding the risk scoring condition according to the World Health Organization criteria, $77.6 \%$, 9.1\%, and $13.3 \%$ were categorized as low-, intermediate-, and high-risk cases. Single therapy with methotrexate was used in $22.8 \%$ of patients and actinomycin-D was planned for $42.3 \%$ whereas $11.0 \%$ and $1.5 \%$ were considered for treatment with the EMA-CO (Etoposide, methotrexate, actinomycin D, cyclophosphamide, vincristine) and EMA-EP (Etoposide methotrexate and actinomycin-D/ etoposide and cisplatin) regimens, respectively. Good response to methotrexate was $66.7 \%$ but it was $83.6 \%$ in the ACT group ( $P$ $=0.001$ ). The resistance to single-agent chemotherapy in low- and intermediate-risk groups was $16 \%$ and $92 \%$, respectively. In addition, $20.2 \%$ of patients in stage one had tumor invasion pattern in the uterus in pretreatment Doppler ultrasonography, but $52 \%$ and $30 \%$ had resistance to chemotherapy treatment in invasive and noninvasive groups, respectively $(P=0.008)$.

Conclusions: In general, due to the high resistance of the intermediate-risk subgroup to a single therapy, a combination therapy may be more useful to treat this disorder. The close association between tumor invasion pattern in the uterus in Doppler ultrasonography and drug resistance can be considered as a new criterion for tumor risk scoring.

Keywords: GTN, Intermediate risk, Chemotherapy resistance
\end{abstract}

\section{Introduction}

Gestational trophoblastic disorders include proliferative disorders that originate from the placental trophoblastic tissue with two none-neoplastic (hydatidiform moles) and neoplastic (gestational trophoblastic neoplasia or GTN) patterns (1). GTN consists of three entities including choriocarcinoma, epithelioid trophoblastic tumor, and placental site trophoblastic tumor (2). In addition, GTN may follow term or preterm pregnancy, molar pregnancy, abortion, or ectopic pregnancy (3). This tumor can be manifested by uterine bleeding or extrauterine hemorrhages $(4,5)$.

Following the molar evacuation, serial serum human chorionic gonadotropin (hCG) levels are measured weekly until it becomes undetectable. Further, postmolar GTN is diagnosed by the International Federation of Gynecology and Obstetrics (FIGO) criteria as follows (6):

- Weekly hCG levels plateau over a three-week period;

- An increase of $>10 \%$ in the hCG level in a two-week duration;
- $\quad$ Existence of $\beta$-HCG six months or more after molar evacuation;

- Existence ofhistological diagnosis of choriocarcinoma. The diagnosis of GTN after a nonmolar pregnancy is evaluated with serum hCG and ultrasound after patients become symptomatic.

For patients with GTN, both a stage and a risk score are assigned before treatment. The staging and scoring system can predict the possibility of resistance to singleagent chemotherapy with methotrexate (MTX) and actinomycin D. One of the main issues in the management of GTN is to investigate the main causes of resistance to common therapeutic regimens. Some previous studies emphasized the importance of the earlier identification of chemotherapy resistance in patients $(7,10)$. Although prognosis in GTN was shown to be excellent, chemotherapy resistance was considered as a challenging matter in the treatment of GTN patients (11).

Considering the above-mentioned explanation, the present study attempted to provide a clear view of GTN

Received 3 January 2019, Accepted 7 May 2019, Available online 20 June 2019

${ }^{1}$ Gynecology Oncology Department, Vali-Asr Hospital, Tehran University of Medical Sciences, Tehran, Iran. ${ }^{2}$ Department of Obstetrics and Gynecology, Faculty of Medicine, Shahid Sadoughi University of Medical Sciences, Yazd, Iran.

*Corresponding Author: Fahimeh Nokhostin, Tel: +983538224000, Email: Fahimeh.nokhostin@yahoo.com 
in ten years in our center with respect to chemotherapy resistance in GTN subgroups.

\section{Materials and Methods}

The protocol of this retrospective cohort study was approved by at Research and Ethical Committees at Tehran University of Medical Sciences (ethical code: IR.TUMS.IKHC.REC.1397.239). Then, the medical records of all patients with the definitive diagnosis of GTN were retrospectively reviewed, who referred to the Department of Gynecology and Oncology of Vali-Asr, Imam Khomeini hospital of Tehran between June 2007 and December 2017. According to Chapman-Davis et al (12), with a $95 \%$ confidence interval and 5\% error, the minimum sample size was 256 . There were 355 medical records of GTN patients who referred to our hospital in 10 years. All these medical records were studied as our sample size. In this study, only the cases suffering from an invasive mole or gestational choriocarcinoma were assessed and thus other types of malignancies including placental site trophoblastic tumor and epithelioid trophoblastic tumor were not included because of the small sample size or information unavailability. The baseline characteristics including age, the antecedent pregnancy, clinical manifestations, and the level of pre-treatment serum $\beta$-hCG, as well as the results of imaging, the site of metastasis, the types of cycles of chemotherapy, and the outcome of surgical management were extracted from the recorded files at the obstetrics and gynecology clinic. In addition, those with incomplete data were collected by telephone follow-ups. After the definitive diagnosis of GTN, all participants referring to the clinic were physically examined and assessed by imaging tools such as chest X-ray, chest CT scan, and pelvic ultrasonography. Further, abdominopelvic CT scan or magnetic resonance imaging was indicated in cases suspected to metastasis or extra-uterine invasion. Then, the patients were staged by the FIGO guidelines $(13,14)$. Furthermore, the World Health Organization (WHO) risk classification rule was employed to determine the risk level as the low (score 0 to 4 ), intermediate (score 5 to 6 ), and high (score $\geq$ 7) risk. The patients were finally treated based on the clinical decision of the physician as medical therapy with methotrexate 30 to $50 \mathrm{mg} / \mathrm{m}^{2}$ intramuscular weekly or actinomycin-D $1.25 \mathrm{mg} / \mathrm{m}^{2}$ to a maximum $2 \mathrm{mg}$ single dose repeated every 14 days for low- and intermediaterisk group. Additionally, EMA/CO (i.e., Etoposide, methotrexate, actinomycin-D, cyclophosphamide, and vincristine) or EMA/EP (a regimen that substitutes cyclophosphamide and vincristine on day 8 with cisplatin and etoposide) chemotherapy regimens for the high-risk group. The patients were followed-up for 3 to 24 months with a mean follow-up time of 12.0 months.

For statistical analysis, the results were reported as mean \pm standard deviation (SD) for quantitative variables and presented by absolute frequencies and percentages for categorical variables. Then, they were compared using the chi-square or Fisher exact tests. The quantitative variables were also compared with the $t$ test or Mann-Whitney $\mathrm{U}$ test. Finally, SPSS, version 16.0 for windows (SPSS Inc., Chicago, IL) was used for data analysis. The $P$ values of 0.05 or less were considered statistically significant.

\section{Results}

Overall, 272 patients were qualified and enrolled in our study. The underlying characteristics of patients are summarized in Table 1. The average age of the participants was $29.19 \pm 7.46$ years ranging from 14 to 53 years and $39.3 \%$ of them were primigravid cases. The most common clinical feature included abnormal uterine bleeding

Table 1. Baseline Characteristics of Study Population

\begin{tabular}{|c|c|}
\hline Variables & \\
\hline Mean age (year), Mean \pm SD & $29.19 \pm 7.46$ \\
\hline \multicolumn{2}{|l|}{ Gravid, No.(\%) } \\
\hline 1 & $107(39.3)$ \\
\hline 2 & $81(29.8)$ \\
\hline 3 & $50(18.4)$ \\
\hline More & $34(12.5)$ \\
\hline \multicolumn{2}{|l|}{ Clinical manifestations, No.(\%) } \\
\hline AUB & $175(64.3)$ \\
\hline Pain & $50(18.4)$ \\
\hline Amenorrhea & 27 (9.9) \\
\hline Nausea & $16(5.9)$ \\
\hline Hyperemesis gravidarum & $16(5.9)$ \\
\hline Hemoptysis & $9(3.3)$ \\
\hline \multicolumn{2}{|l|}{ Pre-treatment $\beta$-HCG, No.(\%) } \\
\hline$<1000$ & $68(25.0)$ \\
\hline $1000-10000$ & $98(36.0)$ \\
\hline $10.000-100000$ & $53(19.5)$ \\
\hline$>100000$ & $53(19.5)$ \\
\hline \multicolumn{2}{|l|}{ Antecedent pregnancy, No.(\%) } \\
\hline Complete mole & $158(58.0)$ \\
\hline Partial mole & $38(14.1)$ \\
\hline Abortion & $59(22.1)$ \\
\hline Term pregnancy & $15(5.5)$ \\
\hline Ectopic pregnancy & $1(0.3)$ \\
\hline \multicolumn{2}{|l|}{ FIGO stage, No.(\%) } \\
\hline I & $198(72.8)$ \\
\hline ॥ & $13(4.8)$ \\
\hline III & $57(21.0)$ \\
\hline IV & $4(1.4)$ \\
\hline \multicolumn{2}{|l|}{ FIGO/WHO score, No.(\%) } \\
\hline 0-4 (low risk) & $211(77.6)$ \\
\hline 5-6 (intermediate risk) & $25(9.1)$ \\
\hline$\geq 7$ (high risk) & $36(13.3)$ \\
\hline Mean number of chemotherapy cycles & $5.0 \pm 1.0$ \\
\hline \multicolumn{2}{|l|}{ Type of surgery, No.(\%) } \\
\hline Evacuation & $47(17.3)$ \\
\hline Hysterectomy & $19(7.0)$ \\
\hline Evacuation and hysterectomy & $3(1.1)$ \\
\hline None & $203(74.6)$ \\
\hline
\end{tabular}

AUB: Abnormal uterine bleeding; hCG: Human chorionic gonadotropin; FIGO: International Federation of Gynecology and Obstetrics; WHO: World health organization. 
(AUB) in $64.3 \%$ of patients, followed by pelvic pain and amenorrhea in $18.4 \%$ and $9.9 \%$ of cases, respectively. Regarding antecedent pregnancy, the complete and partial moles were observed in $58 \%$ and $14.1 \%$, respectively, and abortion was found in $22.1 \%$. The pre-treatment serum $\beta$-hCG level was less than 1000 in $25.0 \%$ while the level of higher than 100000 was detected in $19.5 \%$ of the patients. In addition, $72.8 \%, 4.8 \%, 21 \%$, and $1.4 \%$ of patients were in stages 1, 2, 3, and 4, respectively. As regards the risk scoring condition according to the WHO criteria, $77.6 \%, 9.1 \%$, and $13.3 \%$ of cases were categorized as low, intermediate, and high risk, respectively.

Regarding the patient's stages and risks, several chemotherapy regimens were prescribed for all patients (Table 2). Single therapy with methotrexate was used in $22.8 \%$ of all patients and actinomycin-D was planned for $42.3 \%$ whereas $11.0 \%$ and $1.5 \%$ were considered for treatment with the EMA/CO and EMA/EP regimens, respectively. Good response to methotrexate was $66.7 \%$ but it was $83.6 \%(P=0.001)$ in the ACT group (Table 3$)$. The resistance to single-agent chemotherapy in low- and intermediate-risk groups was $16 \%$ and $92 \%$, respectively. Similarly, resistance to the first-line multi-agent drug in the high-risk group was $8 \%$. Further, $20.2 \%$ of patients in stage 1 had a tumor invasion pattern in the uterus in pretreatment Doppler ultrasonography but $52 \%$ and $30 \%$ of them had resistance to chemotherapy treatment in invasive and noninvasive groups, respectively $(P<0.008)$. For controlling severe vaginal bleeding, evacuation, hysterectomy, as well as hysterectomy and evacuation were scheduled for $17.3 \%, 7.0 \%$, and $1.1 \%$, respectively. However, the non-surgical approach was considered in $74.6 \%$ of patients.

There was a significant difference in the prevalence of clinical symptoms in different tumor stages, indicating that the AUB was the prominent symptom in stages I and II, pain in stage III, along with nausea and amenorrhea in stage IV (Figure 1). The mean number of cycles for chemotherapy did not vary in different stages (stage I: $14.50 \pm 3.54$, stage III: $8.40 \pm 4.45$, stage IV: $8.33 \pm 2.52, P$ $=0.204)$.

\section{Discussion}

GTN affects the women of reproductive age and it is treated by different types of chemotherapy regimens.
Table 3. Comparing Resistance to Single Agent Drug in Low-Risk Patients

\begin{tabular}{lccc}
\hline Drug Response & MTX & ACT & P Value \\
\hline Good response & $66.7 \%$ & $83.6 \%$ & 0.001 \\
Resistance & $33.3 \%$ & $16.4 \%$ & 0.001 \\
\hline
\end{tabular}

Note. MTX: Methotrexate; ACT: Actinomycin-D.

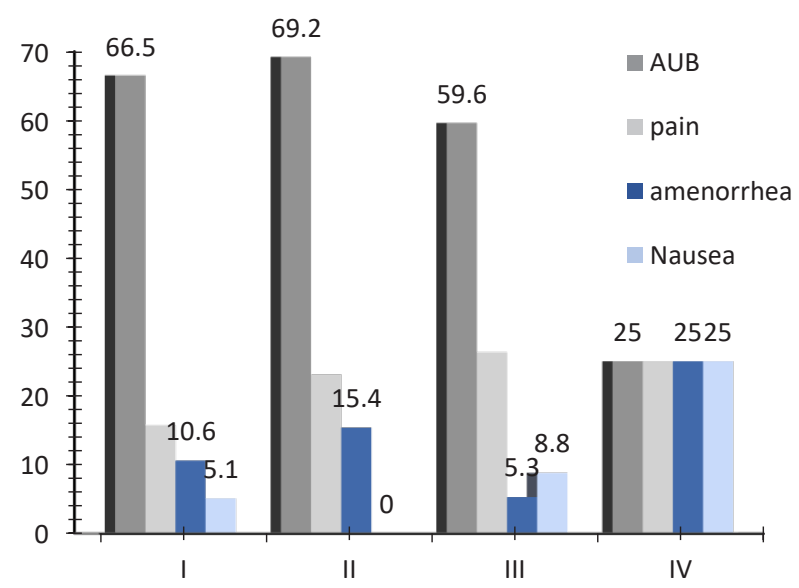

Figure 1. The Frequency of Clinical Symptoms According to Tumor Stage. Note. Horizontal bars: GTN stages (1, 2, 3, \& 4); Vertical bars: Number of patients (the frequency of clinical symptoms).

It can influence future fertility or early menopause of women (15). The main risk factors related to GTN progression include previous molar pregnancy, within the age of 40 years and above, and Asian and American ancestry (16). Unfortunately, no comprehensive view is available with respect to epidemiological aspects, clinical features, and treatment outcomes of patients suffering from gestational trophoblastic tumors in our country. The current study attempted to present a clear view of such neoplasia among our population. In our study, the average age of the participants was 29 years ranging from 14 to 53 years, which is near those of the other studies like Melamed et al (17) and Mayun et al (18) demonstrating a mean age of 24.5 and 25.7, respectively. In our study, complete and partial moles, along with abortion were the most frequent gestational events related to the tumors. Approximately, GTN occurs following molar pregnancy, abortion or ectopic pregnancy, and term or preterm delivery in $50 \%, 25 \%$, and $25 \%$ of patients, respectively $(19,20)$. In our study, complete and partial moles were

Table 2. Chemotherapy Regimens and Stage

\begin{tabular}{|c|c|c|c|c|c|}
\hline Tumor stage & MTX & ACT & EMACO & EMA-EP & Resistant \\
\hline 1 & $54(27 \%)$ & 97 (48\%) & $0(0 \%)$ & $0(0 \%)$ & $47(23 \%)$ \\
\hline II & $2(15 \%)$ & $3(23.1 \%)$ & $7(53 \%)$ & $0(0 \%)$ & $1(7 \%)$ \\
\hline III & $6(10 \%)$ & $15(26 \%)$ & $19(33 \%)$ & $3(5 \%)$ & $14(24 \%)$ \\
\hline IV & $0(0.0)$ & $0(0 \%)$ & $1(25 \%)$ & 1 (25\%) & $2(50 \%)$ \\
\hline Total & $62(22.8 \%)$ & 115 (42.3\%) & 27 (11\%) & $4(1.5 \%)$ & 64 (24\%) \\
\hline
\end{tabular}

Note. MTX: Methotrexate; ACT: Actinomycin-D; EMACO: Etoposide, methotrexate, actinomycin-D, cyclophosphamide, and vincristine; EMA-EP: A regimen that substitutes cyclophosphamide and vincristine on day eight with cisplatin and etoposide. 
revealed in $58 \%$ and $14.1 \%$ of our subjects, respectively. In addition, the most prevalent clinical symptoms included AUB (64.3\%) and pelvic pain (18.4\%). The most common symptom in the study by Suprasert et al (21) was the AUB ( $40 \%$ of patient). It shows that attention to changing the statue of menstruation bleeding is important. The first manifestation in metastatic GTN patients can be bleeding from vital organs such as lung, liver, brain, gastrointestinal tract, and vagina $(22,23)$ but it is less common.

In our survey, about one-third of the patients with gestational trophoblastic tumors faced with raised pretreatment serum $\beta$-hCG level (higher than 10000) and only $19.5 \%$ experienced an increase in the $\beta$-hCG higher than 100000 . The raising serum $\beta$-hCG level can be an important diagnostic finding in patients suffering from gestational trophoblastic tumors. However, very high levels of this serum biomarker are unpredictable so that in our study only one-fifth of the patients demonstrated a rate higher than 100000 .

In our study, good response to methotrexate and ACT in low-risk GTN was $66.7 \%$ and $83.6 \%$, respectively, and overall, the primary remission to single-agent drugs was achieved in $77.6 \%$ of cases. In the study by $\mathrm{Li}$ et al, complete response to ACT in low-risk GTN was $71.1 \%$ (24), which is similar to our results. The most commonly used agents for low-risk GTN were methotrexate and actinomycin-D. At the New England Trophoblastic Disease Center, methotrexate is a first-line drug because of its lower side effect compared to actinomycin-D (25). In a randomized trial of the Gynecologic Oncology Study Group (26), Act-D had a higher complete response rate compared to the MTX in the low-risk GTN (70\% versus $53 \%, P=0.01)$. However, single-agent chemotherapy can be very useful for treating low-risk patients, but as it is well shown, the response to the biweekly actinomycin-D regimen is significantly higher than weekly intramuscular methotrexate. In addition, physicians prefer more to prescribe actinomycin-D than methotrexate in our center. Further, among low- (score 0-4) and intermediaterisk (score 5-6) subgroups, resistance to single-agent chemotherapy was $16 \%$ and $92 \%$, respectively. Thus, the intermediate-risk group probably needs multiagent chemotherapy drugs. According to Mousavi et al, the resistance to single-agent chemotherapy in the intermediate-risk patients was 14 times higher than the low-risk patients (27), which is very close to our finding. Furthermore, Li et al noted that a FIGO score $\geq 5$ is an important factor showing resistance to the ACT (24). Perhaps, a combination therapy can be considered for the patients stratified as the intermediate-risk subgroups, but this suggestion requires more studies in further trials.

Moreimportantly, theinvasive pattern of the uterustumor in stage 1 demonstrated by the Doppler ultrasonography was associated with resistance to therapeutic regimens. Agarwal et al concluded that the uterine artery pulsatility index in Doppler sonography was a good prognostic factor for resistance to MTX (28). Additionally, Li et al showed that the existing invasive uterine lesions were a significant factor in resistance to the ACT (24). Likewise, Akhavan et al found that ultrasonography can be a good diagnostic test for the invasive mole and our finding shows that it can be a good prognostic test for the drug response (29). This novel finding should be more assessed in future studies, but the authors recommended that all patients suspected to gestational trophoblastic tumors should be evaluated with respect to invasive behavior. Moreover, the presence of an invasion pattern can be considered as a new criterion for tumor risk scoring. Our finding reveals that combination therapy is an appropriate treatment for the intermediaterisk subgroup of GTN and Doppler ultrasonography is a good devise to predict chemotherapy resistance although this issue needs further investigation.

The retrospective nature of this study was its main limitation because some file information was incomplete or different physicians visited patients and some of them acted arbitrarily.

\section{Conclusions}

The patients suffering from low-risk GTN responded well to both single therapy with methotrexate or actinomycin-D, but the latter medication was more preferred. Thus, a combination therapy may be more useful for treating this disorder because of the high resistance of the intermediaterisk subgroup to single therapy. Finally, the presence of the invasion pattern can be considered as a new criterion for tumor risk scoring due to a close association between the invasive pattern of the tumor in the uterus in the Doppler ultrasonography and drug resistance.

\section{Conflict of Interests}

Authors declare that they have no conflict of interests.

\section{Financial Support}

None to be declared.

\section{References}

1. Hui P, Baergen R, Cheung A, et al. Gestatinoal trophoblastic disease. In: Kurman RJ, Carcangiu ML, Herrington CS, Young RH, eds. WHO Classification of Tumours of Female Reproductive Organs. 4th ed. Lyon, France: International Agency for Research on Cancer; 2014.

2. Hui P. Gestational Trophoblastic Tumors: A Timely Review of Diagnostic Pathology. Arch Pathol Lab Med. 2019;143(1):65-74. doi:10.5858/arpa.2018-0234-RA

3. Heller DS. Update on the pathology of gestational trophoblastic disease. APMIS. 2018;126(7):647-654. doi:10.1111/apm.12786

4. Duong J, Ghanchi H, Miulli D, Kahlon A. Metastatic Nongestational Choriocarcinoma to the Brain: Case Report and Proposed Treatment Recommendations. World Neurosurg. 2018;115:170-175. doi:10.1016/j. wneu.2018.04.050 
5. McCarthy CM, Unterscheider J, Burke C, Coulter J. Metastatic gestational choriocarcinoma: a masquerader in obstetrics. Ir J Med Sci. 2018;187(1):127-129. doi:10.1007/ s11845-017-1636-6

6. Kohorn EI. The FIGO 2000 staging and risk factor scoring system for gestational trophoblastic neoplasia: a critical analysis. In: Hancock BW, Seckl MJ, Berkowitz RS, Cole LA, eds. Gestational Trophoblastic Neoplasia. 3rd ed. London: ISSTD; 2003.

7. van Trommel NE, Massuger LF, Schijf CP, ten KateBooij MJ, Sweep FC, Thomas CM. Early identification of resistance to first-line single-agent methotrexate in patients with persistent trophoblastic disease. J Clin Oncol. 2006;24(1):52-58. doi:10.1200/jco.2005.03.3043

8. Savage P, Seckl M, Short D. Practical issues in the management of low-risk gestational trophoblast tumors. J Reprod Med. 2008;53(10):774-780.

9. Kerkmeijer LG, Thomas CM, Harvey R, et al. External validation of serum hCG cutoff levels for prediction of resistance to single-agent chemotherapy in patients with persistent trophoblastic disease. $\mathrm{Br} \mathrm{J}$ Cancer. 2009;100(6):979-984. doi:10.1038/sj.bjc.6604849

10. Growdon WB, Wolfberg AJ, Goldstein DP, et al. Evaluating methotrexate treatment in patients with low-risk postmolar gestational trophoblastic neoplasia. Gynecol Oncol. 2009;112(2):353-357. doi:10.1016/j.ygyno.2008.11.003

11. Patel SM, Desai A. Management of drug resistant gestational trophoblastic neoplasia. J Reprod Med. 2010;55(7-8):296300.

12. Chapman-Davis E, Hoekstra AV, Rademaker AW, Schink JC, Lurain JR. Treatment of nonmetastatic and metastatic low-risk gestational trophoblastic neoplasia: factors associated with resistance to single-agent methotrexate chemotherapy. Gynecol Oncol. 2012;125(3):572-575. doi:10.1016/j.ygyno.2012.03.039

13. Shih IM, Kurman RJ. p63 expression is useful in the distinction of epithelioid trophoblastic and placental site trophoblastic tumors by profiling trophoblastic subpopulations. Am J Surg Pathol. 2004;28(9):1177-1183. doi:10.1097/01.pas.0000130325.66448.al

14. Fadare O, Parkash V, Carcangiu ML, Hui P. Epithelioid trophoblastic tumor: clinicopathological features with an emphasis on uterine cervical involvement. Mod Pathol. 2006;19(1):75-82. doi:10.1038/modpathol.3800485

15. Jagtap SV, Aher V, Gadhiya S, Jagtap SS. Gestational trophoblastic disease-clinicopathological study at tertiary care hospital. J Clin Diagn Res. 2017;11(8):EC27-EC30. doi:10.7860/jcdr/2017/27232.10458

16. Cioffi R, Bergamini A, Gadducci A, et al. Reproductive outcomes after gestational trophoblastic neoplasia. A comparison between single-agent and multiagent chemotherapy: retrospective analysis from the MITO-
9 group. Int J Gynecol Cancer. 2018;28(2):332-337. doi:10.1097/igc.0000000000001175

17. Melamed A, Gockley AA, Joseph NT, et al. Effect of race/ ethnicity on risk of complete and partial molar pregnancy after adjustment for age. Gynecol Oncol. 2016;143(1):7376. doi:10.1016/j.ygyno.2016.07.117

18. Mayun A, Rafindadi A, Shehu M. Pathomorphology of molar gestation in Zaria. Niger Med J. 2010;51(1):1-4.

19. American Joint Committee on Cancer. Gestational trophoblastic tumors. In: Amin MB, Edge S, Greene F, et al, eds. AJCC Cancer Staging Manual. 8th ed. New York, NY: Springer; 2017:437-444.

20. FIGO staging for gestational trophoblastic neoplasia 2000. FIGO Oncology Committee. Int J Gynaecol Obstet. 2002;77(3):285-287. doi:10.1016/s0020-7292(02)00063-2

21. Suprasert P, Siriaree S, Manopunya M. Outcomes of metastatic gestational trophoblastic neoplasia: fourteen year experience from a northern Thailand tertiary care center. Asian Pac J Cancer Prev. 2016;17(3):1357-1362. doi:10.7314/APJCP.2016.17.3.1357

22. Soper JT. Gestational trophoblastic disease. Obstet Gynecol. 2006;108(1):176-187. doi:10.1097/01. AOG.0000224697.31138.a1

23. Seckl MJ, Fisher RA, Salerno G, et al. Choriocarcinoma and partial hydatidiform moles. Lancet. 2000;356(9223):36-39. doi:10.1016/s0140-6736(00)02432-6

24. Li L, Wan X, Feng F, et al. Pulse actinomycin D as first-line treatment of low-risk post-molar non-choriocarcinoma gestational trophoblastic neoplasia. BMC Cancer. 2018;18(1):585. doi:10.1186/s12885-018-4512-5

25. Berkowitz RS, Goldstein DP, Bernstein MR. Ten year's experience with methotrexate and folinic acid as primary therapy for gestational trophoblastic disease. Gynecol Oncol. 1986;23(1):111-118. doi:10.1016/0090-8258(86)90123-x

26. Osborne RJ, Filiaci V, Schink JC, et al. Phase III trial of weekly methotrexate or pulsed dactinomycin for low-risk gestational trophoblastic neoplasia: a gynecologic oncology group study. J Clin Oncol. 2011;29(7):825-831. doi:10.1200/ jco.2010.30.4386

27. Mousavi AS, Zamani A, Khorasanizadeh F, Gilani MM, Zendehdel K. Resistance to single-agent chemotherapy and its risk factors in low-risk gestational trophoblastic neoplasms. J Obstet Gynaecol Res. 2015;41(5):776-783. doi:10.1111/jog.12613

28. Agarwal R, Strickland S, McNeish IA, et al. Doppler ultrasonography of the uterine artery and the response to chemotherapy in patients with gestational trophoblastic tumors. Clin Cancer Res. 2002;8(5):1142-1147.

29. Akhavan S, Mousavi AS, Modares Gilani M, Alibakhshi A, Rahmani M, Karim N. The Value of Ultrasonography in Predicting Invasive Mole. J Obstet Gynecol Cancer Res. 2016;1(2):e7965. doi:10.17795/ojcr-7965 